\title{
7-Hydroxyquinoline.( $\left.\mathrm{NH}_{3}\right)_{3}$ : A Model for Excited State H-Atom Transfer Along an Ammonia Wire
}

\author{
Christian Tanner*, Carine Manca, and Samuel Leutwyler
}

\begin{abstract}
The 7-hydroxyquinoline $\left(\mathrm{NH}_{3}\right)_{3}$ cluster has been used as a model to study excited state $\mathrm{H}$-atom transfer along a hydrogen-bonded $\mathrm{NH}_{3} \cdots \mathrm{NH}_{3} \cdots \mathrm{NH}_{3}$ wire. Excitation of the supersonically cooled cluster to its vibrationless $\mathrm{S}_{1}$ state produces no reaction, whereas excitation of ammonia-wire vibrations induces fluorescence of the excited 7-ketoquinoline. $\left(\mathrm{NH}_{3}\right)_{3}$ cluster, revealing the excited state enol $\rightarrow$ keto tautomerization. This reaction is assumed to occur via successive $\mathrm{H}$ transfers along the ammonia wire in a Grotthus-like process. Ab initio calculations of the reaction path show that proton and electron movement along the wire are closely coupled. The rate-controlling $\mathrm{S}_{1}$ state barriers arise from crossing of $a \pi \pi^{\star}$ and a Rydberg-type $\pi \sigma^{\star}$ electronic excited state.
\end{abstract}

Keywords: Ammonia wire $\cdot$ CIS and CASSCF calculations · Excited state $\cdot$ Hydrogen transfer $\cdot$ Molecular beam

Proton and hydrogen transfer are among the fundamental and best-studied reactions in biology, especially proton transport through membrane ion channels ('proton wires'). Many transmembrane proteins create, control or utilize proton gradients across biological membranes. An example of a so-called 'proton pump' is the photosynthetic reaction center of Rhodobacter sphaeroides, which contains a chain of water molecules [1]. Direct experimental observation of the motion of protons along a wire is difficult, because of the short times, microscopic length scales, and solvent fluctuations involved. Thus we focus on simple model systems in order to understand the most basic aspects of proton conduction along hydrogen-bonded wires.

7-Hydroxyquinoline (7HQ) is an aromatic molecule which combines an $\mathrm{O}-\mathrm{H}$

\footnotetext{
${ }^{\star}$ Correspondence: C. Tanner

Universität Bern

Department für Chemie und Biochemie

Freiestrasse 3

$\mathrm{CH}-3000$ Bern 9

Tel.: +41316314238

Fax: +41316313994

E-Mail: christian.tanner@iac.unibe.ch
}

group as $\mathrm{H}$ donor with a quinolinic $\mathrm{N}$ acceptor site (Fig. 1a). This molecule is employed as a framework for a hydrogenbonded wire consisting of three $\mathrm{NH}_{3}$ molecules, the two hydrogen bonding sites being spaced far enough apart to stretch the wire. A different way of looking at the proton transfer process is that the enol $\rightarrow$ keto tautomerization of 7-hydroxyquinoline is mediated by the wire (the keto form is shown in Fig. 1b). Our recent laser spectroscopic experiments on jet-cooled ammonia clusters $7 \mathrm{HQ} \cdot\left(\mathrm{NH}_{3}\right)_{\mathrm{n}}$ have allowed size-dependent studies on the proton transfer process and we have shown that for $n=5$ or 6 , ground-state proton transfer with formation of zwitterions occurs [2][3]. DFT calculations predict Grotthus-type proton transfer steps along the wire [2]. For the $\mathrm{n}=$ 3 cluster, no reaction has been found in the ground state. Indeed, $a b$ initio calculations predict the enol form to be $36 \mathrm{~kJ} \mathrm{~mol}^{-1}$ more stable than the keto in the gas phase; the latter is not observed in our supersonic molecular beam experiments at $5 \mathrm{~K}$.

In the $S_{1}$ state, the keto form is predicted to be $46 \mathrm{~kJ} \mathrm{~mol}^{-1}$ more stable than the enol. The SCF and CIS geometry optimizations in the ground and the excited state allows the estimation of the geometry changes upon $\mathrm{S}_{1} \leftarrow \mathrm{S}_{0}$ excitation: All four hydrogen bonds contract, as indicated in Fig. $1 \mathrm{a}$, by -0.03 to $-0.08 \AA$, the larger decreases occurring at the ends of the wire. In parallel, the intramolecular $\mathrm{O}-\mathrm{H}$ bond is predicted to lengthen by $+0.01 \AA$. These geometric changes upon excitation of 7HQ are intuitively expected to favor the $\mathrm{enol} \rightarrow$ keto reaction.

The $\mathrm{S}_{1} \leftarrow \mathrm{S}_{0}$ two-color resonant 2-photon ionization (2C-R2PI) spectrum of 7HQ $\left(\mathrm{NH}_{3}\right)_{3}$ is shown in Fig. 2a: it is mainly composed of sharp bands (FWHM of 1-2 $\left.\mathrm{cm}^{-1}\right)$ in the range $28800-29000 \mathrm{~cm}^{-1}$. The most intense band at $28798.4 \mathrm{~cm}^{-1}$ is assigned to the electronic origin. The band at $+13 \mathrm{~cm}^{-1}$ is an ammonia wire bending mode and the stronger bands in the range +125 to $+200 \mathrm{~cm}^{-1}$ correspond to ammonia wire stretching vibrations; for more details, see [4].

The UV-UV depletion spectrum shown in Fig. 2b is obtained by scanning a holeburning laser over the same spectral region, 300 ns before the 2C-R2PI measurement, thereby depopulating the vibrational ground state. The resulting UV-UV depletion spectrum shows the same components as the 2C-R2PI spectrum from the origin up to $200 \mathrm{~cm}^{-1}$. Contrary to the $2 \mathrm{C}-\mathrm{R} 2 \mathrm{PI}$ spectrum, it exhibits further vibronic bands far above $200 \mathrm{~cm}^{-1}$. Complementary measurements of UV fluorescence (not shown here) present a similar falloff at $\sim 200 \mathrm{~cm}^{-1}$ above the electronic origin. The loss of both the 2C-R2PI and UV fluorescence signals reveals the loss of the excited state enol $7 \mathrm{HQ}^{*} \cdot\left(\mathrm{NH}_{3}\right)_{3}$ cluster population and prove the onset of a fast process at $200 \mathrm{~cm}^{-1} \mathrm{ex}$ cess energy in the $S_{1}$ state.

Concurrent to the loss of the UV fluorescence of $7 \mathrm{HQ}^{*} \cdot\left(\mathrm{NH}_{3}\right)_{3}$, we observe an 


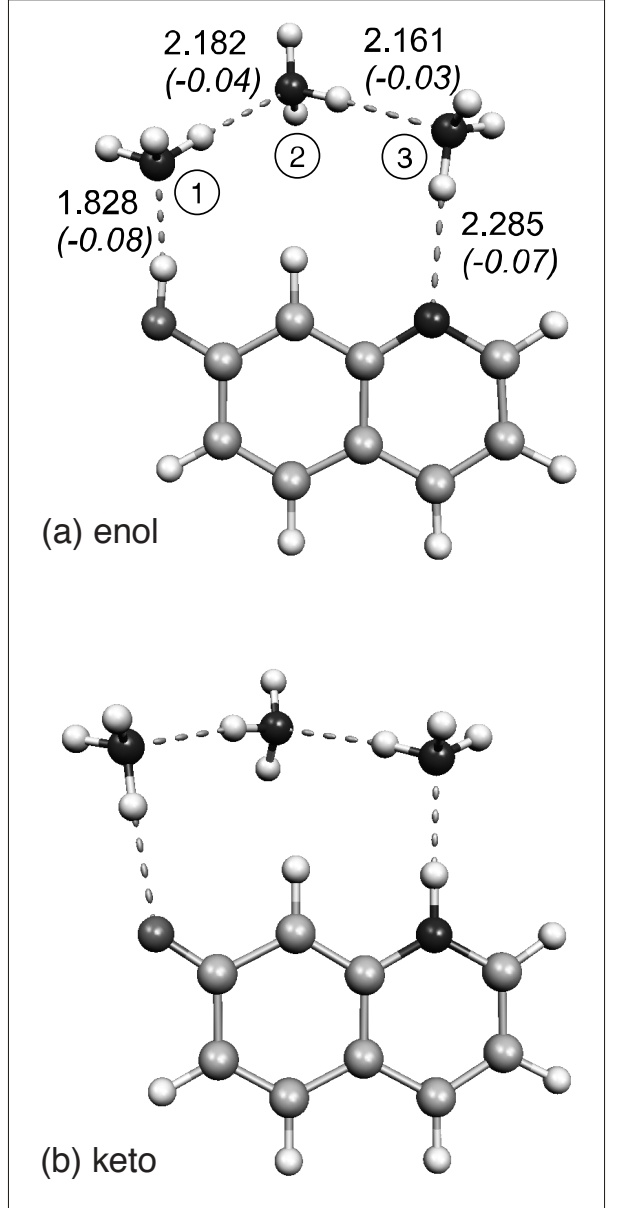

Fig. 1. Calculated stable structures of the enol (a) and the keto (b) tautomeric forms of the 7hydroxyquinoline $7 \mathrm{HQ} \cdot\left(\mathrm{NH}_{3}\right)_{3}$ cluster. For the enol form, hydrogen bond lengths between $\mathrm{H}$ and heavy atom in the ground state are indicated in $\AA$, changes upon excitation are given in parentheses.

increase in yellow fluorescence. Such fluorescence has already been observed in bulk protic solvents and has been assigned to the $\mathrm{S}_{1}$ state 7-ketoquinoline (7KQ*) tautomer [5]. Fluorescence excitation spectra (scanning the excitation laser and monitoring the maximum of the yellow fluorescence at $18360 \mathrm{~cm}^{-1}$, Fig. 2c) exhibit the same characteristic vibronic band pattern of $7 \mathrm{HQ} \cdot\left(\mathrm{NH}_{3}\right)_{3}$ as in the UV-UV depletion, starting from the two highest vibronic bands observed in the 2C-R2PI spectrum, $200 \mathrm{~cm}^{-1}$ above the origin (the remaining fluorescence background arises from larger $7 \mathrm{HQ} \cdot\left(\mathrm{NH}_{3}\right)_{\mathrm{n}}$ clusters $)$. In other words, the excitation of $7 \mathrm{HQ} \cdot\left(\mathrm{NH}_{3}\right)_{3}$ clusters $\geq 200$ $\mathrm{cm}^{-1}$ above the electronic origin leads to formation and fluorescence emission of the $7 \mathrm{KQ}^{*} \cdot\left(\mathrm{NH}_{3}\right)_{3}$ cluster.

We used CIS and CASSCF excited state calculations in order to interpret the experiments and propose a mechanism. We assume that the excited state enol $\rightarrow$ keto tautomerization observed involves $\mathrm{H}^{+}$or $\mathrm{H}$ translocation along the $\left(\mathrm{NH}_{3}\right)_{3}$ wire to the quinolinic $\mathrm{N}$ atom, in a Grotthus-type reaction [6]. The upper part of Fig. 3 shows the schematic $S_{1}$ potential connecting all the fully optimized CIS stationary structures obtained. The ground-state enol form has been taken as reference for these calculations and the excited-state potential has been arbitrarily offset to match the experimental value of the electronic origin. We have found three minima between the enol and the keto form: these correspond to the $\mathrm{H}$-atom transferred intermediates denoted HT1, HT2, and HT3 respectively. These are separated by four transition states labeled $\mathrm{TS}_{\mathrm{E} / 1}, \mathrm{TS}_{1 / 2}, \mathrm{TS}_{2 / 3}$, and $\mathrm{TS}_{3 / \mathrm{K}}$. Fig. 4 shows the first reaction step between enol and $\mathrm{HT}$ 1, involving an $\mathrm{H}$ transfer from the hydroxyl group of 7HQ to the first ammonia molecule. The three other steps can be obtained by the corresponding $\mathrm{H}$ transfer through the ammonia wire to the quinolinic N.

Further CASSCF calculations were performed with the cluster constrained to $C_{s}$ symmetry to clarify the key points of the reaction. The $S_{1}$ and $S_{2}$ potentials obtained with these calculations are schematically shown in Fig. 3 (dotted and dashed lines). The calculations predict that the $\mathrm{S}_{1} \leftarrow \mathrm{S}_{0}$ excitation occurs dominantly from the highest occupied $\pi$ molecular orbital on $7 \mathrm{HQ}$. The enol $\mathrm{S}_{1}$ state is a $\pi \pi^{*}\left(\mathrm{~A}^{\prime}\right)$ excita- tion localized on $7 \mathrm{HQ}$, whereas the $\mathrm{S}_{2}$ state is mainly a $\pi \sigma^{*}\left(A^{\prime \prime}\right)$ Rydberg-type excitation. Due to different spatial symmetry, these states can cross along the reaction path between the enol and the HT1 forms and create a conical intersection. In nonplanar geometries, such an intersection is forbidden, leading to repulsion of the two states in an adiabatic description. The barrier at $\mathrm{TS}_{\mathrm{E} / 1}$ of the CIS calculations originates from the crossing of the $\pi \pi^{*}$ and $\pi \sigma^{*}$ states, indicating a qualitative change of the excited state character. The CIS calculated electronic barrier height is $3720 \mathrm{~cm}^{-1}(44.5$ $\mathrm{kJ} \mathrm{mol}^{-1}$ ) which is probably too high since the CIS method is known to typically overestimate such barriers [7]. The enol $\rightarrow$ HTI step is predicted to be exoergic by about $2600 \mathrm{~cm}^{-1}\left(31.1 \mathrm{~kJ} \mathrm{~mol}^{-1}\right)$, which provides the driving force of the reaction. The last barrier at $\mathrm{TS}_{3 / \mathrm{K}}$ in CIS calculations originates from a reverse crossing between the $\pi \sigma^{*}$ and $\pi \pi^{*}$ states as diagnosed in the CASSCF calculations, leading to the $\pi \pi^{*}$ excited $7 \mathrm{KQ}^{*} \cdot\left(\mathrm{NH}_{3}\right)_{3}$ tautomer. According to the CIS calculation, the HT1, HT2, HT3, and $7 \mathrm{KQ}$ forms lie close in energy; once the system passes through the $\mathrm{TS}_{\mathrm{E} / 1}$ barrier, the two subsequent barriers are surmounted. The last barrier $\mathrm{TS}_{3 / \mathrm{K}}$ between the HT3 and

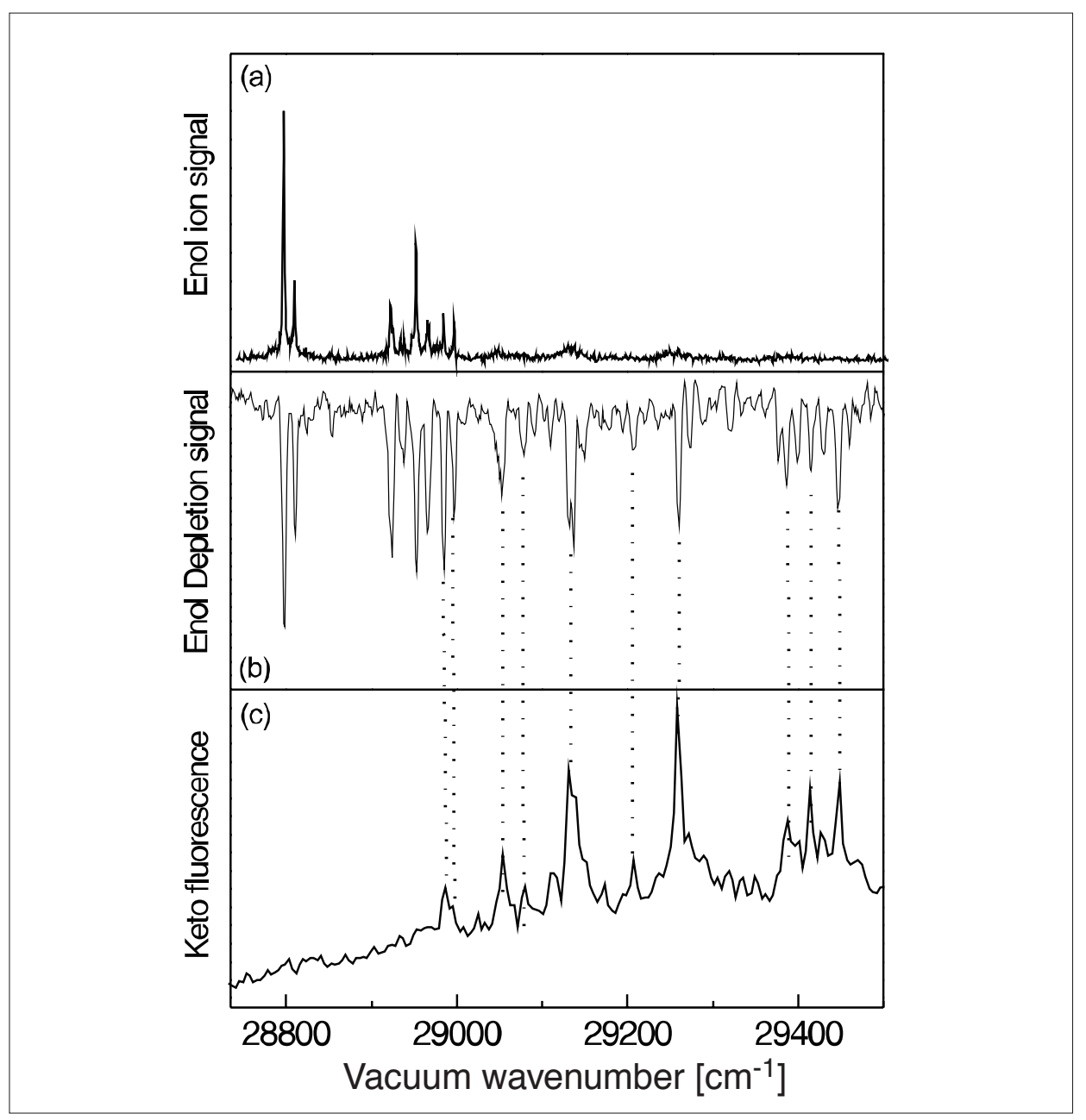

Fig 2. a: 2-Color Resonant 2-Photon Ionization spectrum of $7 \mathrm{HQ} \cdot\left(\mathrm{NH}_{3}\right)_{3}$. b: UV-UV depletion spectrum of $7 \mathrm{HQ} \cdot\left(\mathrm{NH}_{3}\right)_{3}$. c: Excitation fluorescence of $7 \mathrm{HQ} \cdot\left(\mathrm{NH}_{3}\right)_{3}$ at $18360 \mathrm{~cm}^{-1}$. 


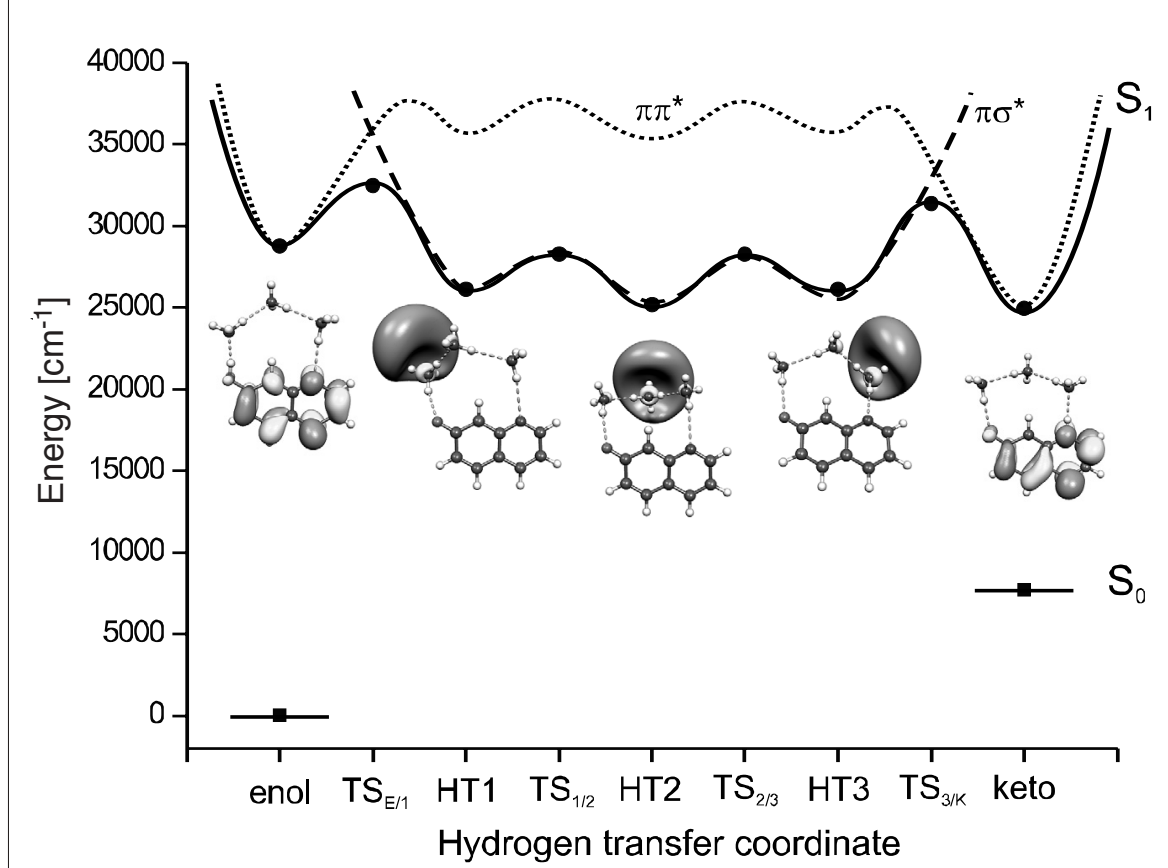

Fig 3. Excited state $\left(\mathrm{S}_{1}\right)$ enol $\rightarrow$ keto conversion pathways of $7 \mathrm{HQ} \cdot\left(\mathrm{NH}_{3}\right)_{3}$. The full line indicates the $\mathrm{CIS}$ potential. The dotted and dashed lines schematically indicate the $\pi \pi^{\star}$ and $\pi \sigma^{*}$ states calculated with the CASSCF method. The energies of the enol and the keto form in the ground state $\left(\mathrm{S}_{0}\right)$ are also indicated. The unoccupied molecular orbital that contributes dominantly to the $S_{1}$ state wave function is shown for the enol, the keto and the three intermediate minima.

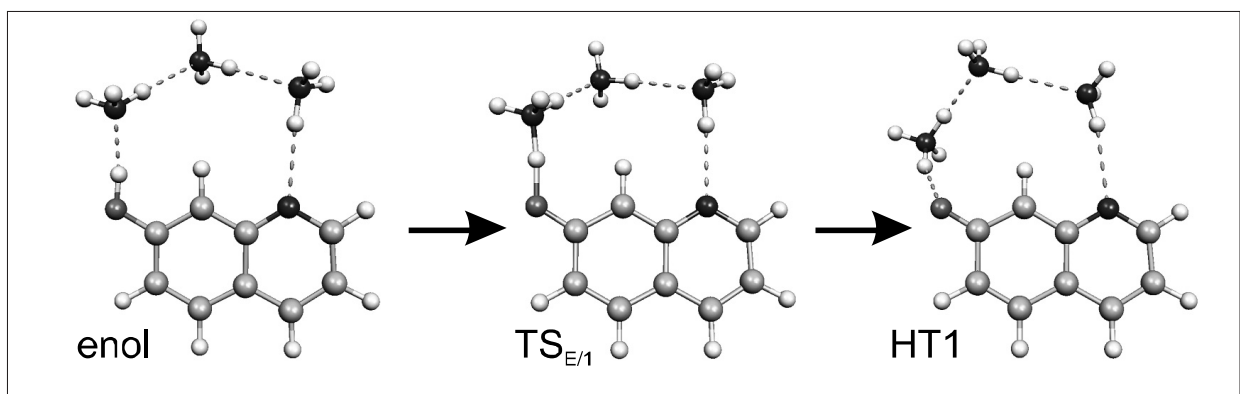

Fig 4. The reactant, transition state and product structures for the first step of the proposed $\mathrm{H}$-atom transfer reaction between the enol and the $\mathrm{HT} 1$ form, $\mathrm{TS}_{\mathrm{E} / 1}$ being the transition state.

$7 \mathrm{KQ}$ forms is calculated to be nearly as high as $\mathrm{TS}_{\mathrm{E} / 1}$. The $\mathrm{S}_{1} \leftarrow \mathrm{S}_{0}$ energy difference for $7 \mathrm{KQ}$ after $\mathrm{S}_{1}$ state translation (see above) is predicted to be $18,020 \mathrm{~cm}^{-1}$, in agreement with experimental fluorescence maximum at $18,360 \mathrm{~cm}^{-1}$.

The configuration contributing dominantly to the excited wave function involves promotion of an electron from the highest occupied $\pi$ orbital of $7 \mathrm{HQ}$. The change in character of the electron-accepting antibonding orbital has already been detailed elsewhere [8]. Briefly, for the enol, this is the antibonding $\pi^{*}$ orbital of $7 \mathrm{HQ}$, whereas for HT1 it is an antibonding $\sigma^{*}$ orbital on the newly generated $\mathrm{NH}_{4}$ moiety (Fig. 3). A transfer of 0.9 electron from the $7 \mathrm{HQ}$ moiety to $\mathrm{NH}_{3}(1)$ occurs simultaneously with this change of excited-state character: the proton transfer becomes a $\mathrm{H}$ atom transfer in the vicinity of $\mathrm{TS}_{\mathrm{E} / 1}$. The lowest excited state retains the $\sigma^{*}$ character along the translocation coordinate up to $\mathrm{TS}_{3 / \mathrm{K}}$ and the $\sigma^{*}$ orbital accompanies the $\mathrm{H}$ atom (Fig. 3). A reverse $\sigma^{*} \rightarrow \pi^{*}$ state switching occurs near $\mathrm{TS}_{3 / \mathrm{K}}$; the $7 \mathrm{KQ}$ orbital is a $\pi^{*}$ orbital similar to that of the enol.

We emphasize the correlation between the $\pi^{*} \rightarrow \sigma^{*} \rightarrow \pi^{*}$ crossovers and the changes of oscillator strength for the $\mathrm{S}_{1} \rightarrow$ $\mathrm{S}_{0}$ transition: According to $C_{s}$ symmetry selection rules, fluorescence is allowed from the $\pi \pi^{*}$ but forbidden from the $\pi \sigma^{*}$ state and this selection rule holds approximately also for non $C_{s}$ geometry here: The calculated $S_{1} \rightarrow S_{0}$ oscillator strength for $7 \mathrm{HQ}$ and $7 \mathrm{KQ}$ like states are $\mathrm{f}=0.193$ and 0.294 respectively, whereas for HT1 to HT3 states they are $f=0.001$ to 0.004 . This difference explains why the HT1, HT2 and HT3 forms have much longer radiative lifetimes and why no fluorescence is observed from these intermediates in our experiments.

The role of low-lying $\pi \sigma^{*}$ Rydbergtype electronic states for excited state hydrogen transfer of heteroaromatic mole- cules has been studied for phenol, indole, 1naphthol: $\left(\mathrm{NH}_{3}\right)_{\mathrm{n}}$ and phenol- $\left(\mathrm{NH}_{3}\right)_{\mathrm{n}}$ clusters [7][9]. For all these systems, the lowest $\pi \sigma^{*}$ excited states are dissociative along the $\mathrm{N}-\mathrm{H}$ or $\mathrm{O}-\mathrm{H}$ bond stretching coordinates. Concerning the $7 \mathrm{HQ} \cdot\left(\mathrm{NH}_{3}\right)_{3}$, it is worth noting that the $\pi \sigma^{*}$ state does not lead to cluster dissociation but to $\mathrm{H}$ atom transfers from the O-H group of 7HQ along the ammonia wire.

Summarizing, we have shown that $\mathrm{H}$ atom transfer can be induced along the ammonia wire connected to 7-hydroxyquinoline in the $\mathrm{S}_{1}$ excited state. Additional excitation of $S_{1}$ state ammonia wire vibrations is necessary to activate the reaction $(2.5 \mathrm{~kJ}$ $\mathrm{mol}^{-1}$ experimentally). Ab initio calculations predict a crossing of $\pi \pi^{*}$ and $\pi \sigma^{*}$ potential energy curves along the $\mathrm{H}$ atom transfer coordinate that creates an initial barrier of $\sim 44 \mathrm{~kJ} \mathrm{~mol}^{-1}$. At least the first reaction step involves quantum tunneling from the ground state of the $\mathrm{O}-\mathrm{H}$ mode. It is exothermic by about $30 \mathrm{~kJ} \mathrm{~mol}^{-1}$, which provides the driving force for the subsequent reaction steps.

Received: January 19, 2004

[1] U. Ermler, G. Fritzsch, S. Buchanan, H. Michel, Structure 1994, 2, 925.

[2] M. Meuwly, A Bach, S. Leutwyler, J. Am. Chem. Soc. 2001, 123, 11446.

[3] A. Bach, C. Tanner, C. Manca, H.-M. Frey, S. Leutwyler, J. Chem. Phys. 2003, 119 , 5933.

[4] C. Manca, C. Tanner, S. Coussan, A. Bach, S. Leutwyler, J. Chem. Phys., submitted.

[5] S.F. Mason, J. Philip, B.E. Smith, J. Chem. Soc. A. 1968, 3051; S. Schulman, Q. Fernando, Tetrahedron 1968, 24, 1777; K. Tokumura, M. Ioth, J. Phys. Chem. 1984, 88, 3921; E. Bardez, Israel J. Chemistry 1999, 39, 319 .

[6] D. Borgis, J. Hynes, Chem. Phys. 1993, 170, 315; N. Agmon, Chem. Phys. Lett. 1995, 244, 456; D. Marx. M.E. Tuckerman, J. Hutter, M. Parrinello, Nature 1999, 397, 601; P.L. Geissler, C. Dellago, D. Chandler, J. Hutter, M. Parrinello, Science 2001, 291, 2121; D. Lu, G.A. Voth, J. Am. Chem. Soc. 1998, 120, 4006; R. Vuilleumier, D. Borgis, J. Phys. Chem. B 1998, 102, 4261; H.S. Mei, M.E. Tuckerman, D.E. Sagnella, M.L. Klein, J. Phys. Chem. $B$ 1998, 102, 10446 .

[7] A.L. Sobolewski, W. Domcke, Phys. Chem. Chem. Phys. 1999, 1, 3065; A.L. Sobolewski, W. Domcke, J. Chem. Phys. A 2001, 105, 9275; A.L. Sobolewski, W. Domcke, C. Dedonder-Lardeux, C. Jouvet, Phys. Chem. Chem. Phys. 2002, 4, 1093.

[8] C. Tanner, C. Manca, S. Leutwyler, Science 2003, 302, 1736.

[9] A.L. Sobolewski, W. Domcke, J. Chem. Phys. A 1999, 103, 4494; O. David, C. Dedonder-Lardeux, C. Jouvet, Int. Rev. Phys. Chem. 2002, 21, 499. 